\title{
Modelos de Regressão para Estimativa do Volume de Fuste e Ramos da Espécie Brachystegia gossweilerii Hutch. \& Burt Davy, na Floresta do Miombo da Comuna do Cuima (Huambo- Angola)
}

\author{
André Ndjamba ${ }^{1}$, Yordan Pérez ${ }^{2}$, Verena Cárdenas ${ }^{3}$, Célcio Luamba \\ e Abilio Lalengue ${ }^{5}$
}

Sumário. O presente trabalho teve como finalidade ajustar modelos matemáticos para estimar o volume do fuste e ramos para a espécie Brachystegia gossweilerii Hutch. E Burt Davy. Para o efeito, foram derrubadas e rigorosamente cubadas 53 árvores da espécie acima mencionada. A área de estudo corresponde a floresta de Miombo da Aldeia Calombo, comuna do Cuima, Município da Caála, Província do Huambo. Os dados, obtidos das árvores foram processados, utilizando para o efeito o programa SPSS versão 15.0 para Windows. Os modelos de melhor ajuste para estimativa do volume do fuste com casca é o modelo aritmético de entrada simples $\log V r t=-1,058+1,023 \log _{g t}+0,485 * 1 / \log _{g t}^{2}$, enquanto para os ramos foi o modelo $V r t=0,297 * g t^{1,987}$.

Palavras-chave: Equações; volume; Miombo; massa; Huambo.

${ }^{1}$ Mestre em gestão de espécies da flora e fauna ameaçadas de extinção. Docente da Faculdade de Ciências Agrárias (FCA) da Universidade José Eduardo dos Santos (UJES). Angola.

E-mail: ndjamba72@hotmail.com

2Doutor em Ciências Florestais. Universidade de Guantánamo. República Popular de Cuba.

E-mail: yordanlp@cug.co.cu

${ }^{3}$ Doutora em Ciências Agrícolas. Investigadora Titular do Instituto de Ciência Animal. República Popular de Cuba. E-mail: vtorres@ica.co.cu

${ }^{4}$ Engenheiro Florestal. Estagiário da FCA da UJES. Angola. E-mail: celcioluambagaspar@live.com

${ }^{5}$ Mestre em ciências florestais. Docente da FCA da UJES. Angola. E-mail: abmalengue@gmail.com 
Regression Models for Estimating the Volume of Stem and Branches of the Species Brachystegia gossweilerii Hutch. \& Burt Davy, in the Miombo Forest of The Commune of Cuima (Huambo-Angola)

Abstract: The present work aimed to adjust mathematical models to estimate the volume of the stem and branches for the species Brachystegia gossweilerii Hutch. \& Burt Davy. To this end, 53 trees of the aforementioned species were felled and rigorously cubed. The study area corresponds to the Miombo forest of Village Calombo, commune of Cuima, Municipality of Caála, Province of Huambo. The data obtained from the trees were processed using SPSS version 15.0 for Windows. The best fit models for estimating the volume of the stem with bark is the simple entry arithmetic model, $\log V r t=-1,058+1,023 \log _{g t}+0,485 * 1 / \log _{g t}^{2}$ while for the branches it was the model $V r t=0,297 * g t^{1,987}$.

Key words: Equations; volume; Miombo; mass; Huambo.

Modèles de Régression pour l'Estimation du Volume de Fút et des Branches de l'Espèce Brachystegia gossweilerii Hutch. \& Burt Davy, Dans la Forêt de Miombo de la Commune de Cuima (Huambo-Angola)

Résumé: Le présent travail visait à ajuster des modèles mathématiques pour estimer le volume du fút et des branches pour l'espèce Brachystegia gossweilerii Hutch. \& Burt Davy. À cette fin, 53 arbres des espèces susmentionnées ont été abattus et rigoureusement coupés en cubes. La zone d'étude correspond à la forêt Miombo du Village Calombo, commune de Cuima, Commune de Caála, Province de Huambo. Les données obtenues à partir des arbres ont été traitées à l'aide de SPSS version 15.0 pour Windows. Le modèle le mieux adapté pour estimer le volume du fút avec l'écorce est le modèle arithmétique à entrée $\log V r t=-1,058+1,023 \log _{g t}+0,485 * 1 / \log _{g t}^{2}$, tandis que pour les branches c'était le modele $V r t=0,297 * g t^{1,987}$.

Mots-clés: Équations; volume; Miombo; masse; Huambo 


\section{Introdução}

Angola é um dos países da África subsaariana que possui uma das maiores extensões e diversidade florestais. A sua extensão territorial é de $1.246 .700 \mathrm{Km}^{2}$, $43,3 \%$ dos quais estão cobertos por florestas. Os seus recursos florestais têm sido considerados como capital natural disponível que, com baixos níveis de investimento poderão gerar benefícios económicos, sociais e ambientais para o estado e a sociedade, através da sua exploração e utilização sustentável (MINADER, 2011).

As formações florestais são bastante diferenciadas, sendo que a floresta do Miombo com uma área que corresponde a aproximadamente $244.000 \mathrm{Km}^{2}$, da superfície florestal de Angola é o tipo de floresta mais extenso. Neste tipo particular de floresta predominam as espécies dos géneros Brachystegia, Julbernardia e Isoberlinia da família das Fabaceae, reveste-se de grande importância para a vida das pessoas no meio rural e urbano por serem fonte de energia na forma de lenha e carvão, fornecerem materiais de construção, produtos alimentares, medicinais, de entre outros (FREIRE, 2010).

De salientar que em Angola cerca de $60 \%$ da população vive no meio rural e tem na lenha, no carvão, na carne de caça, no mel, nos insectos e nos frutos silvestres as suas principais fontes de subsistência e renda, os quais são providos principalmente pela floresta do Miombo (MINADER, 2011).

Estudos profundos sobre as espécies do Miombo de Angola e mais concretamente sobre equações de volume para construção de tabelas dendrométricas são escassos.

Segundo FREIRE (2010) o valor potencial da maior parte das espécies vegetais dos ecossistemas florestais de Angola é pouco conhecido, e muitas que parecem insignificantes sob a óptica urbana podem ter uma importância vital para as comunidades rurais, como as plantas medicinais e os alimentos obtidos da floresta.

A província do Huambo é uma das que fazem parte da região central do país onde as suas florestas típicas são as do Miombo. Nesta, assim como na maior parte das províncias de Angola, a exploração florestal não é feita de forma adequada, o que tem elevado o risco para a manutenção e renovação deste importante recurso. A expansão da agricultura itinerante, a fixação humana espontânea, o desmatamento para área de criação de gado, o corte para lenha e carvão, a exploração madeireira e a falta de instrumentos necessários para auxiliar na planificação e gestão das florestas são muitas das causas que 
contribuem para a redução das superfícies florestais e assim como o empobrecimento destas.

A Brachystegia gossweilerii Hutch. \& Burt Davy pertence a um dos géneros mais representativos do Miombo. O estudo desta espécie poderá fornecer subsídios muito importantes para a compreensão do comportamento das demais espécies deste género e, por conseguinte, para a formulação de planos de maneio florestal mais adequados à realidade.

Esta espécie apresenta um alto valor para a o fabrico de carvão vegetal que é uma das principais actividades realizadas pela população da aldeia Calombo, melhorando assim a situação socioeconómica da população que reside nesta área. Por este motivo necessita de um estudo de modelos matemáticos de regressão de volume não só para o fuste, mas também para os ramos.

O volume individual da árvore em pé é uma das informações de importância capital para o conhecimento do potencial disponível em um povoamento florestal, uma vez que fornece informação para a análise do potencial produtivo da floresta (THOMAS et al., 2016). Identificar e seleccionar parâmetros biométricos da árvore ou povoamento florestal para quantificar e estimar a estrutura da mesma, e desta forma poder auxiliar os processos de tomada de decisão na gestão da floresta é uma tarefa essencial e fundamental.

Segundo LEITE and RESENDE (2010), o volume é estimado através de modelos ajustados de regressão, podendo ser linear (simples ou múltipla) ou não linear. Assim sendo, o presente estudo teve como objectivo ajustar modelos matemáticos para estimar o volume do fuste e ramos para a espécie Brachystégia gossweilerii Hutch. \& Burt Davy, na floresta do Miombo da comuna do Cuima, município da Caála (Huambo-Angola).

\section{Material e métodos}

\section{Caracterização da área de estudo}

A área de estudo localiza-se na aldeia Calombo, comuna do Cuima, município de Caála, na província do Huambo em Angola. Está situada entre as coordenadas geográficas $13^{\circ} 14^{\prime} 40^{\prime \prime}$ Sul e $15^{\circ} 38^{\prime} 29^{\prime \prime}$ Leste. Tem uma área de aproximadamente $3680 \mathrm{~km}^{2}$ e no seu interior está a aldeia com cerca de $373 \mathrm{mil}$ habitantes. O Município da Caála localiza-se na parte central da província do Huambo tendo como limites a Norte os municípios da Ekunha a Este o município de Huambo, a Sul o município de Chipindo (Província da Huíla), e a 
Oeste os Municípios de Longonjo e Caconda. O município da Caála é constituído pelas comunas de Caála, Cuima (área de estudo), Kalenga e Katata. A floresta de Miombo presente na área de estudo é de domínio público pertencente à aldeia, caracterizada por estar constituída na sua totalidade por árvores naturais em forma de manchas florestais da formação de Brachystegia ssp., com temperaturas médias anuais que variam entre 19 e $20^{\circ} \mathrm{C}$, podendo considerar-se como um clima temperado quente, com valores de precipitações que oscilam desde os $1100 \mathrm{~mm}$ a S-SW até um pouco acima dos $1400 \mathrm{~mm}$ no topo planáltico Centro-Oeste. O período chuvoso tem início nos finais de Setembro e prolonga-se até meados ou mesmo finais de Abril. O clima do Huambo é quente e temperado. O verão é muito mais chuvoso que o inverno. De acordo com o sistema Köppen-Geiger é classificado de Cwb (DINIZ, 2006).
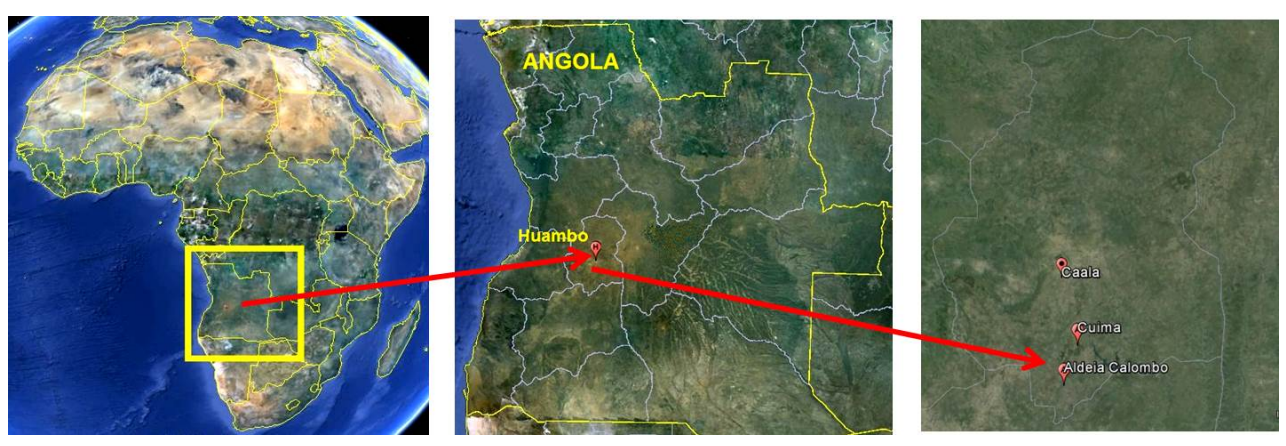

Figura 1- Localização da área de estudo. Fonte: Google Earth

Obtenção dos dados

Foram levantadas parcelas circulares de $500 \mathrm{~m}^{2}$ com 12,62 m de raio, conforme mostra a Figura 2, parcelas estas distribuídas mediante uma amostragem aleatória estratificada. Para a formação dos estratos considerou-se como variável de interesse o número de árvores por hectare. Portanto, no cálculo da área da unidade de amostragem corrigiu-se este valor de raio de $12,62 \mathrm{~m}$ para o valor que corresponde à sua medida num plano horizontal. Para o efeito foi utilizada a fórmula:

$$
R_{h}=R_{i} \cos \alpha
$$

Onde:

$R_{h}=$ Raio na linha horizontal;

$R_{i}=$ Raio na linha em declive;

$\alpha=$ Valor da pendente medida em graus; 


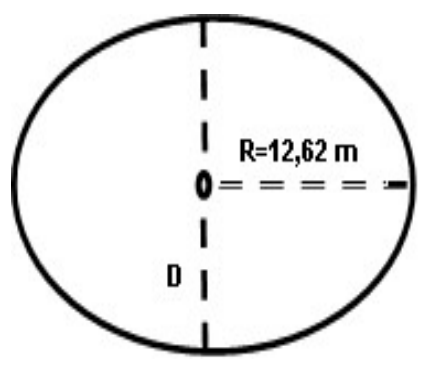

Figura 2 - Dimensão da parcela de amostragem

Determinou-se o tamanho da amostra utilizando parcelas de área fixa como unidades de amostragem de tal maneira que nestas amostras estejam representadas todas nas áreas do Miombo.

\section{Tamanho da amostra}

Efetuou-se uma pré-amostragem com 29 parcelas para o cálculo do número de parcelas, onde se utilizou como variável de interesse o número de árvores por hectare. As parcelas foram distribuídas por meio de amostragem aleatória, estratificada, em que o limite de erro utilizado é de 15\% com uma probabilidade de $95 \%$. Para o efeito, a população foi dividida em quatro estratos: Estrato I - de 0 até 300 árvores/hectare; Estrato II - de 301 a 600 árvores/hectare; Estrato III de 601 a 900 árvores/hectare; Estrato IV - mais de 901 árvores/hectare (Quadro 1).

Em seguida determinou-se o tamanho da população da região em estudo que corresponde a 928,4 ha de área florestal pertencente à aldeia Calombo onde se realizou a investigação, isto é, o número total de parcelas de $500 \mathrm{~m}^{2}$, ou seja 0,05 ha que há na área de investigação, para o qual utilizou-se a fórmula: $N=\frac{A}{a}$ Onde:

$A$ = área total da população $(928,4$ ha);

$a=$ área da parcela amostral 0,05 ha. 
Quadro 1 - Número de parcelas por estrato com seu respectivo número de árvores por hectare a partir do inventário prévio

\begin{tabular}{|c|c|c|c|c|}
\hline Parcelas & Estrato I & Estrato II & Estrato III & Estrato IV \\
\hline 1 & 0,0 & 480,0 & 620,0 & 3200,0 \\
\hline 2 & 0,0 & 460,0 & 680,0 & 1740,0 \\
\hline 3 & 0,0 & 540,0 & 740,0 & 1860,0 \\
\hline 4 & 40,0 & 450,0 & 800,0 & 2560,0 \\
\hline 5 & 0,0 & & & \\
\hline 6 & 0,0 & & & \\
\hline 7 & 0,0 & & & \\
\hline 8 & 0,0 & & & \\
\hline 9 & 60,0 & & & \\
\hline 10 & 0,0 & & & \\
\hline 11 & 0,0 & & & \\
\hline 12 & 0,0 & & & \\
\hline 13 & 160,0 & & & \\
\hline 14 & 220,0 & & & \\
\hline 15 & 260,0 & & & \\
\hline 16 & 300,0 & & & \\
\hline 17 & 220,0 & & & \\
\hline
\end{tabular}

Para a selecção da fórmula com vista ao cálculo do tamanho da amostra, avaliou-se se a população objecto de investigação era finita ou infinita por meio da execução das hipóteses seguintes:

Se $1-f \geq 0,98$ a população seria infinita, a fórmula para usar seria:

$$
n=\frac{t^{2} \sum_{n=1}^{L} W_{h} s_{h}^{2}}{E^{2}}
$$

Se $1-f \geq 0,98$ a população seria finita, então a fórmula a usar seria:

$$
n=\frac{t^{2} \sum_{h=1}^{L} W_{h} s_{h}^{2}}{E^{2}+t^{2} \sum_{h=1}^{L} \frac{W_{h} s_{h}^{2}}{N}} \text { onde: } E=\frac{L E \%}{100} * \bar{x}_{s t}
$$

Onde:

$f=\frac{n}{N}=$ fracção de amostragem da população; $L=$ número de estratos;

$h=$ estrato; $N_{h}=$ número potencial de unidades do estrato $h$ na população;

$W_{h}=\frac{N_{h}}{N}=$ Proporção do estrato $h$ na população; 


$$
\begin{aligned}
& s_{h}^{2}=\frac{\sum_{i=1}^{n_{h}}\left(X_{i h}-\bar{x}_{h}\right)^{2}}{n_{h}-1}=\text { Variância por estrato; } \\
& E=\text { erro ou a precisão desejada; } \\
& t=\text { Valor de } \mathrm{t} \text { de Student para } 95 \% \text { de probabilidade. }
\end{aligned}
$$

Além disso, foram calculadas outras variáveis estatísticas tais como:

Média por estrato: $\bar{x}_{h}=\frac{\sum_{i=1}^{n_{h}} X_{i h}}{n_{h}}$

Média estratificada: $\bar{x}_{s t}=\frac{\sum_{h=1}^{L} n_{h} \bar{x}_{h}}{n}=\sum_{h=1}^{L} W_{h} \bar{x}_{h}$

Variância estratificada: $s_{s t}^{2}=\sum_{h=1}^{L} W_{h} s_{h}^{2}$;

Erro padrão: $s_{\bar{x}(s t)}=\sqrt{\sum_{h=1}^{L} W_{h}^{2} \frac{s_{h}^{2}}{n_{h}}\left(1-f_{n}\right)}$

Erro da amostragem:

a) Absoluto: $E_{a}= \pm t s_{\bar{x}(s t)}$

b) Relactivo: $E_{r}= \pm \frac{t s_{\bar{x}(s t)}}{\bar{x}_{s t}} * 100$

Intensidade da amostragem por estrato: $n_{h}=\frac{N_{h}}{N} * n=W_{h} * n$

Os principais índices dendrométricos registados por classes diamétricas foram:

- média da altura $(\bar{h})$,

- média de diâmetro $(\bar{d})$,

- média da altura comercial $\left(\bar{h}_{c}\right)$,

- área basal total por hectare (G/ha),

- volume total por hectare $\left(\mathrm{V}_{\mathrm{t}} / \mathrm{ha}\right)$,

- volume comercial por hectare $\left(\mathrm{V}_{\mathrm{c}} / \mathrm{ha}\right) \mathrm{e}$

- número de árvores por hectare (N/ha).

Mediram-se os diâmetros das árvores a altura de 0,30 e 1,30 m acima do solo e o diâmetro médio, isto para as árvores da espécie em estudo, para avaliar o seu grau de ocupação. As alturas daquelas árvores cuja leitura, no aparelho de medição, foi dificultada por questões relacionadas com o clima ou densidade excessiva foram estimadas. Os índices dendrométricos foram agrupados como se mostra no Quadro 2. 
PRODAN et al., (1997) afirmam que para a construção de tabelas dendrométricas, o total de árvores para a construção de tabelas dendrométricas, contrariamente ao que acontece quando se deseja seleccionar uma amostra para calcular um certo parâmetro, não existe no caso de regressões uma expressão explícita para determinar o número de árvores tipo necessárias. Portanto, quando maior for o número de árvores tipo, mais precisa será a estimativa.

Os principais índices dendrométricos registados por classe diamétrica são apresentados no Quadro 2.

Quadro 2 - Médias gerais dos principais índices dendrométricos da espécie em cada classe diamétrica

\begin{tabular}{|c|c|c|c|c|c|c|}
\hline CD & Intervalo & Ni/ha & D1,30 & D0,30 & Ht & Hc \\
\hline 2 & $1-2,9$ & 83 & 2.3 & 3.4 & 2.4 & 1.5 \\
\hline 4 & $3-4,9$ & 168 & 3.925 & 5.050 & 3.486 & 1.784 \\
\hline 6 & $5-6,9$ & 182 & 5 & 8 & 5 & 2 \\
\hline 8 & $7-8,9$ & 201 & 7.653 & 9.643 & 5.978 & 3.072 \\
\hline 10 & $9-10,9$ & 133 & 9.762 & 12.074 & 6.663 & 2.912 \\
\hline 12 & $11-12,9$ & 82 & 11.697 & 13.503 & 7.452 & 2.818 \\
\hline 14 & $13-14,9$ & 55 & 13.458 & 15.851 & 8.118 & 2.713 \\
\hline 16 & $15-16,9$ & 32 & 15.106 & 18.025 & 9.041 & 2.681 \\
\hline 18 & $17-18,9$ & 42 & 17.7 & 19.25 & 10.183 & 2.952 \\
\hline 20 & $19-20,9$ & 20 & 19 & 19.5 & 8 & 2.9 \\
\hline 22 & $21-22,9$ & 20 & 21 & 22 & 10 & 3 \\
\hline 24 & $23-24,9$ & 20 & 24.2 & 22 & 9 & 3 \\
\hline 30 & $29-30,9$ & 20 & 29.55 & 30.85 & 12.75 & 4.9 \\
\hline Total & & 1060 & 13.9 & 15.3 & 7.5 & 2.8 \\
\hline
\end{tabular}

$\mathrm{CD}=$ Classe Diamétrica; Ni/ha = Número de Árvores por hectare; D1,30 = Diámetro a 1,30 m do solo; $\mathrm{D} 0,30=$ Diámetro a $0,30 \mathrm{~m}$ do solo; $\mathrm{Ht}=$ Altura total; $\mathrm{Hc}=$ Altura comercial.

Como se pode observar na tabela acima, a CD 2 apresenta um Ni/ha de apenas 83, considerado baixo em comparação com as CD imediatamente subsequentes. Este valor deve-se ao facto de se ter definido, por ocasião do levantamento dos dados, de registrar-se apenas as plantas com diâmetros iguais ou superiores a $2 \mathrm{~cm}$, isto devido a confusão que se estava tendo com as espécies não arbóreas ou arbustivas.

Pode-se constatar ainda na referida tabela que a Ht da CD 20, 22 e 24 é menor que das $\mathrm{CD}$ anteriores e posteriores. Tais valores devem-se a acção humana, isto é, tais árvores foram encontradas numa zona em as populações dedicam-se a actividade apícola, tendo por costume o corte de parte da copa das árvores. 
Determinação da área basal por hectare e por classe diamétrica

Para o cálculo do volume, deve-se pôr em evidência a área basal das árvores ou de uma floresta, por ser uma variável com um indicador dendrométrico importante para definir o estado e a capacidade de rendimento florestal.

Para a determinação da área basal por hectare $(\mathrm{G} / \mathrm{ha})$ calcularam-se as áreas basais de cada árvore presente nas parcelas de amostragem com base no diâmetro medido com uma fita diamétrica e através da seguinte fórmula:

$$
G / h a=F \sum_{i=1}^{m} g_{i}=F \sum_{i=1}^{m} \frac{\pi}{4} d_{i}^{2}
$$

Onde:

$G / h a$ = área basal por hectare em $\mathrm{m}^{2}$;

$d_{i}=$ diâmetro médio de cada árvore;

$g_{i}=$ área basal média de cada árvore;

$m$ = número total de árvores na parcela;

$F=\frac{A}{a}=$ factor de proporcionalidade para converter os valores da parcela para valores por hectare $\left(10000 \mathrm{~m}^{2}\right)$

$a$ = área da parcela em hectare ou $\mathrm{m}^{2}$.

Logo a seguir calculou-se a área basal por classe diamétrica que foi determinada mediante a seguinte fórmula:

$$
G_{i}=\frac{\pi}{4} \bar{d}_{i}^{2} * n_{i}
$$

Onde:

$G_{i}=$ área basal da classe diamétrica;

$\bar{d}_{i}=$ média do diâmetro das árvores na classe diamétrica;

$n_{i}=$ número de árvores na classe diamétrica.

Determinação do volume por hectare e por classe diamétrica

Para o cálculo do volume por hectare $(\mathrm{V} / \mathrm{ha})$ utilizou-se a seguinte fórmula:

$$
V / h a=F\left(\sum_{i=1}^{m} v_{i}\right) \text { ou } V / h a=F\left(\sum_{i=1}^{m} \frac{\pi}{4} d_{i}^{2} * h_{i} * f_{i}\right)={ }_{F}\left(\sum_{i=1}^{m} g_{i} * h_{i} * f_{i}\right)
$$

Onde:

$v_{i}=$ volume de cada árvore;

$h_{i}=$ altura estimada de cada árvore;

$f=$ fator volumétrico de cada árvore. 
Como para a espécie investigada, não se conhece exactamente o valor de $\left(f_{i}\right)$ utilizou-se então para a estimativa do volume $\left(v_{i}\right)$ a tabela de coeficientes mórficos empíricos $\left(f_{e}\right)$ e a fórmula, recomendada no Manual para a Ordenação das florestas em Cuba, ou seja: $v_{i}=g_{i} *\left(h_{i}+3\right) * f_{e}$ e também $v_{i}=\frac{\pi}{4} d_{i}^{2} *\left(h_{i}+3\right) * f_{e}$.

O cálculo do volume por classes diamétricas realizou-se mediante a seguinte fórmula:

$$
V_{i}=\left[\frac{\pi}{4} \bar{d}_{i}^{2} *\left(\bar{h}_{i}+3\right) * f_{e}\right] * n_{i}
$$

Onde:

$$
\begin{aligned}
& V_{i}=\text { volume da classe diamétrica; } \\
& \bar{d}_{i}=\text { diâmetro médio da classe diamétrica; } \\
& \bar{h}_{i}=\text { médias das alturas da classe diamétrica; } \\
& n_{i}=\text { número de árvores da classe diamétrica. }
\end{aligned}
$$

Recolha de dados de campo para tabelas de volume

Para a determinação das tabelas de volume foram seleccionadas e derrubadas 53 árvores, nas quais foram medidos os diâmetros da base, diâmetro a $0,30 \mathrm{~m}$, diâmetro a $1,30 \mathrm{~m}$, diâmetro à metade do fuste, diâmetro da extremidade mais fina do fuste e diâmetro dos ramos. Dividiu-se todas as árvores em secções de $50 \mathrm{~cm}$ tanto para o fuste como os ramos, mediu-se o diâmetro da base, do meio e do topo de cada secção. Tais dados foram processados com auxílio do software estatístico SPSS versão 15.0 mediante o qual realizou- se uma análise de regressão para o fuste e ramos.

Calculou-se o volume das diferentes secções do fuste e dos ramos mediante a cubicagem rigorosa de Newton:

$$
V_{1}=\frac{1}{6}\left(g_{i}+4 g m+g_{i+1}\right) * L
$$

Onde:

$V_{1}=$ Volume das secções;

$g_{i}=$ área da secção do extremo maior;

$\mathrm{g}_{i+1}=$ área da secção do extremo menor;

$g_{m}=$ Área da secção da metade do comprimento;

$L=$ comprimento da secção. 
64 Ndjamba, A., et al.

Selecção dos modelos de regressão para o volume

Para a determinação do modelo de melhor ajuste com vista a estimava do volume do fuste e dos ramos, foram testados 20 modelos ( 5 de entrada simples, 8 de dupla entrada e 7 de tripla entrada).

Estes modelos foram testados com diferentes variáveis independentes, na maioria dos casos, aquelas que mais fortemente se correlacionaram na análise de correlação de Pearson. Entre os modelos testados para fuste e ramos, os modelos cúbicos foram ensaiados para diâmetros com casca.

Para a determinação de um modelo único para o fuste e ramos, primeiramente foi necessário construir uma base de dados, em cada caso, com os dados da espécie e logo realizou-se um teste dos melhores dez modelos ajustados individualmente para a espécie. Para o fuste foram ajustados 20 modelos e para os ramos 20.

Comprovou-se a fiabilidade das estatísticas para o volume mediante o erro padrão da estimativa, ou coeficiente de correlação e de determinação e realizouse uma análise das capacidades preditivas e bondade de ajuste dados pela Diferença Agregada (DA) e Raiz do Erro Quadrático Médio (REQM), respectivamente, com objectivo de escolher o modelo de melhor confiabilidade.

A selecção da equação de volume foi feita de acordo com a metodologia proposta por SALAS (2002) e LORES (2012).

A classificação do modelo de melhor ajuste foi indicada a correspondente equação ajustada que apresentou o menor valor do REQM calculado para toda amostra, ou menor valor da média e desvio padrão do REQM nas classes de validação. De igual modo determinou-se o melhor ajuste indicado pela equação que apresentou o DA mais próximo a zero, calculada para toda a amostra, ou menor valor da média em valor absoluto e desvio padrão da DA nas classes de validação.

\section{Resultados e discussão}

\section{Principais variáveis dendrométricas}

Mediante a utilização do programa Microsoft Excel 2003 realizaram-se os cálculos das principais variáveis dendrométricas para a espécie investigada, como se mostra no Quadro 3. 
Quadro 3 - Média dos índices dendrométricos totais

\begin{tabular}{|c|c|c|c|c|c|c|}
\hline Ni/ha & $\begin{array}{c}\bar{D}_{1,30} \\
\text { cm /ha }\end{array}$ & $\begin{array}{c}\bar{D}_{0,30} \\
\text { cm/ha }\end{array}$ & $\begin{array}{l}\bar{H} \mathbf{t} \\
\mathbf{m}\end{array}$ & $\begin{array}{c}\bar{H} \mathbf{c} \\
\mathbf{~ m}\end{array}$ & $\begin{array}{c}\bar{H} \mathbf{d} \\
\mathbf{m}\end{array}$ & $\begin{array}{c}\bar{G}_{1,30} \\
\mathbf{m}^{2} / \mathbf{h a}\end{array}$ \\
\hline 1060 & 13,9 & 15,3 & 7,5 & 2,8 & 6,4 & 26,0 \\
\hline $\bar{G}_{0,30} \mathrm{~m}^{2} / \mathrm{ha}$ & $\begin{array}{l}\bar{V} \mathbf{i t}_{1,30} \\
\text { m3}^{3} / \mathbf{h a}\end{array}$ & $\begin{array}{l}\bar{V} \mathbf{i t}_{0,30} \\
\mathbf{m}^{3} / \mathbf{h a}\end{array}$ & $\begin{array}{l}\bar{V} \mathbf{i c}_{1,30} \\
\mathbf{m}^{3} / \mathbf{h a}\end{array}$ & $\begin{array}{l}\bar{V} \mathbf{i c}_{0,30} \\
\mathbf{m}^{3} / \mathbf{h a}\end{array}$ & $\begin{array}{c}\mathrm{nVi}_{1,30} \\
\mathrm{~m}^{3}\end{array}$ & $\begin{array}{c}\mathbf{n V i}_{0,30} \\
\mathbf{m}^{3}\end{array}$ \\
\hline 34,35 & 21,62 & 23,54 & 22,46 & 15,85 & 50,84 & 59,31 \\
\hline
\end{tabular}

$\mathrm{Ni} /$ ha - número de árvores por hectare; $\mathrm{D}_{1,30}$ - diâmetro a 1,30 $\mathrm{m}$ de altura $(\mathrm{em} \mathrm{cm}) ; \mathrm{D}_{0,30 \text { - diâmetro a }}$ 1,30 m de altura (em $\mathrm{cm})$; Ht - altura total da árvore (em m); Hc - altura comercial (em m); Hd - altura dominante (em m); G1,30 - área basal a 1,30 m (em m²); $\mathrm{G}_{0,30}$-área basal a 0,30 m (em m²); Vit $_{1,30}$ - volume total a $1,30 \mathrm{~m}\left(\mathrm{em} \mathrm{m}^{3}\right) ;$ Vit 0,30 - volume total a $0,30 \mathrm{~m}\left(\mathrm{em} \mathrm{m}^{3}\right) ; V^{2} \mathrm{Vic}_{1,30}$ - volume comercial a $1,30 \mathrm{~m}\left(\mathrm{em} \mathrm{m}^{3}\right) ; V i_{0,30}$ - volume total a $0,30 \mathrm{~m}\left(\mathrm{em} \mathrm{m}^{3}\right) ; \mathrm{nVi}_{1,30}$ - volume total em todas classes diamétricas a $1,30 \mathrm{~m}\left(\mathrm{em} \mathrm{m}^{3}\right) ; \mathrm{nVi}_{0,3}$ volume total em todas classes diamétricas a $0,30 \mathrm{~m}(\mathrm{em}$ $\left.\mathrm{m}^{3}\right)$.

Como se pode observar no Quadro 3, os dados colhidos à altura de $0,30 \mathrm{~m}$ acima do solo apresentam bons resultados, assim nos pode garantir que, a recolha de dados para a planificação, gestão e maneio das florestas do Miombo, os dados podem ser retirados a esta altura.

Todos estes índices dendrométricos foram calculados para diâmetros a altura de 1,30 m do solo entre 5 a $25 \mathrm{~cm}$, onde se observou que na área de estudo existem poucos indivíduos por hectare com diâmetros maiores de $25 \mathrm{~cm}$. Valores iguais a estes obtiveram JAY (2014) em um estudo sobre a eficiência do método de Bitterlich na avaliação de parâmetros dendrométricos na formação florestal do Miombo e por MALENGUE (2014) em um estudo sobre avaliação do potencial de produtos florestais não madeireiros na área florestal de Calombo, Cuima. O que confirma que nas florestas irregulares, como as do Miombo, as parcelas que têm maior número árvores apresentam diâmetros inferiores.

Modelos para equação de volume do fuste e ramos

- Modelos de regressão de volume para o fuste

Um dos argumentos pelos quais se decidiu aplicar a fórmula de Newton é descrito por CAILLIEZ (1980) e realçado por COBAS (2013) que faz uma análise das quatro formas geométricas gerais a que se podem corresponder o fuste ou as distintas partes do fuste das árvores: cilindro, paraboloide, cone e neloide, onde a fórmula de Huber subestima o volume real para a forma de cone e neloide e a fórmula de Smalian sobrestima o volume real para estas mesmas formas, mas 
ambas fórmulas são exactas para o cilindro e paraboloide e afirma que a fórmula de Newton é eficiente para qualquer das formas geométricas do fuste.

Com os dados das 53 árvores derrubadas elaborou-se a matriz de correlação das variáveis para o fuste com o volume real total (Vrt) como variável dependente com respeito às variáveis independentes: $d_{b}$ - diâmetro da base (em $\mathrm{cm}) ; \mathrm{d}_{0,30}$ - diâmetro a altura de $0,30 \mathrm{~m}(\mathrm{em} \mathrm{cm}) ; \mathrm{d}_{1,30}$ - diâmetro a altura de $1,30 \mathrm{~m}$ $(\mathrm{em} \mathrm{cm}) ; \mathrm{d}_{1 / 2}$ - diâmetro médio (em cm); Ht - altura total (em m); Hc - altura comercial (em m); Cc- comprimento da copa (em m); Lc - largura da copa (em $\mathrm{m}) ; \mathrm{Gt}$ - área basal total $\left(\mathrm{em} \mathrm{m}^{2}\right) ; \mathrm{G}_{1,30}$ - área basal a altura de $1,30 \mathrm{~m}\left(\mathrm{em} \mathrm{m}^{2}\right)$ e Df - diâmetro da parte mais fina do fuste (em $\mathrm{cm}$ ) (Quadro 4).

Quadro 4 - Matriz de correlação de Pearson entre as variáveis dendrométricas para o fuste de Brachystegia gossweilerii Hutch. \& Burt Davy

\begin{tabular}{|c|c|c|c|c|c|c|c|c|}
\hline & VrT & Db & D 0,30 & D1,3 & D1/2 & Gt & G1,3 & Df \\
\hline VrT & 1 & $0,867^{* *}$ & $0,845^{* *}$ & $0,755^{* *}$ & $0,696^{* *}$ & $0,966^{* *}$ & $0,748^{* *}$ & $0,684^{* *}$ \\
\hline Db & & 1 & $0,963^{* *}$ & $0,863^{* *}$ & $0,769^{* *}$ & $0,821^{* *}$ & $0,857^{* *}$ & $0,831^{* *}$ \\
\hline D 0,30 & & & 1 & $0,800^{* *}$ & $0,668^{* *}$ & $0,797^{* *}$ & $0,793^{* *}$ & $0,789^{* *}$ \\
\hline D1,3 & & & & 1 & $0,760^{* *}$ & $0,719^{* *}$ & $0,980^{* *}$ & $0,856^{* *}$ \\
\hline D1/2 & & & & & 1 & $0,674^{* *}$ & $0,747^{* *}$ & $0,797^{* *}$ \\
\hline Gt & & & & & & 1 & $0,707^{* *}$ & $0,641^{* *}$ \\
\hline G1,3 & & & & & & & 1 & $0,882^{* *}$ \\
\hline Df & & & & & & & & 1 \\
\hline
\end{tabular}

** A correlação é significativa a nível 0,01 (bilateral).

No Quadro 5 são apresentados os modelos que melhor se ajustaram com base nas análises de bondade de ajuste e as capacidades preditivas com o Vrt como variável dependente. Estes modelos foram testados apenas para as variáveis que apresentaram forte correlação.

Ressalta-se ainda no mesmo quadro que dos dez modelos de melhor ajuste e capacidades preditivas foi o número 1 que melhor resultado apresentou. Este resultado confirma os modelos de LOETSCH, et al. (1973) citado por LORES (2012) que afirmam ainda que as tabelas de volume em função do diâmetro e a altura produzem estimativas mais exactas.

Estes resultados diferem de PECE et al. (2002) citado por COBAS (2013) quando determinaram os melhores modelos de ajustes para a tabela de volume de simples entrada em álamos da zona de rego de Santiago de Estero, Argentina que foi o modelo de dupla entrada de Schumacher Hall. Salienta-se ainda que, 
difere dos modelos propostos em Cuba por PEÑALVER (1991), PADILLA (1999), ZALDÍVAR (2001) para plantação de Eucaliptus sp, Pinus tropicalis, Talipariti elatum respectivamente e por Ares (1999) para Pinus tropicalis e Pinus caribaea em floresta natural, os quais encontraram o modelo logarítmico de Spurr. Também difere de HENRY (2002) citado por LORES (2012) que igualmente encontrou o modelo logarítmico de Suprr com diferentes valores nos coeficientes, com o diâmetro na base como variável dependente, foi o de melhor ajuste para um grupo de espécies latifólias nas áreas cenagosas de Ciénaga de Zapata, Matanzas, onde uma dessas espécies é precisamente Calophyllum antillanum Britton. Assim o mesmo difere também de MACHADO (2003), o qual afirmou que o modelo de melhor ajuste foi o logarítmico de SPURR (1952) de dupla entrada, para as espécies Calophyllum utile, Manilkara albescens, Tabebuia dubia y Sloanea curatellifolia, nestes mesmos tipos de florestas.

Quadro 5 - Melhores dez modelos para o fuste

\begin{tabular}{|c|c|c|c|c|c|c|c|}
\hline \multirow{2}{*}{$\mathbf{N}^{\mathbf{o}}$} & \multirow{2}{*}{ V. Ind. } & \multicolumn{6}{|c|}{ Modelos de melhor ajuste para fuste com $V_{\text {rt }}$} \\
\hline & & $\mathbf{R}$ & $\mathbf{R}^{2}$ & Sx & REQM & DA & Equação \\
\hline 5 & LogGt & 0,989 & 0,977 & 0,138 & 0,000 & 0,018 & $\log V r t=-1,058+1,023 \log _{g t}+0,485 * 1 / \log _{g t}^{2}$ \\
\hline 3 & Gt & 0,988 & 0,977 & 0,139 & 0,000 & 0,019 & $\log V r t=-1,316+0,966 * \log g t$ \\
\hline 4 & Gt & 0,969 & 0,939 & 0,007 & 0,000 & 0,000 & $V r t=-0,010+0,158 g t+0,082 g t^{05}$ \\
\hline 2 & Gt & 0,969 & 0,938 & 0,055 & 0,007 & 0,000 & $V r t=0,001 * g t^{1,633}$ \\
\hline 1 & $\log d b$ & 0,966 & 0,933 & 0,008 & 0,000 & 0,000 & $V r t=0,003+(0,262 * G t)$ \\
\hline 17 & $\log d b$ & 0,930 & 0,866 & 0,340 & 0,000 & 0,109 & $\log V r t=-8,902+1,692 \log d_{b}+0,206 \log +0,436 \log d_{1 / 2}$ \\
\hline 17 & $\log \mathrm{d}_{0,30}$ & 0,930 & 0,864 & 0,342 & 0,000 & 0,110 & $\log V r t=-8,470+0,407 \log d_{0,30}+1,119 \log +0,819 \log d_{1 / 2}$ \\
\hline 17 & $\log d b$ & 0,929 & 0,861 & 0,347 & 0,000 & 1,114 & $\log V r t=-9,592+2,227 \log d_{b}+0,495 \log +0,209 \log d_{f}$ \\
\hline 17 & $\log d b$ & 0,927 & 0,860 & 0,347 & 0,000 & 0,114 & $\log V r t=-9,274+1,597 \log d_{b}+0,506 \log h+0,344 \log d$ \\
\hline 17 & $\log d b$ & 0,927 & 0,859 & 0,349 & 0,000 & 0,115 & $\log V r t=-9,196+1,851 \log d_{b}+0,380 \log +0,166 \log d_{1,30}$ \\
\hline
\end{tabular}

A Figura 3 mostra o grau de correspondências da equação do modelo proposto $\log V r t=-1,058+1,023 \log _{g t}+0,485 * 1 / \log _{g t}^{2}$ com uma tendência conveniente, onde não se obteve diferenças significativas entre o volume estimado e o volume real para uma significação de 0,996 o que garante fiabilidade na aplicação prática deste modelo. 


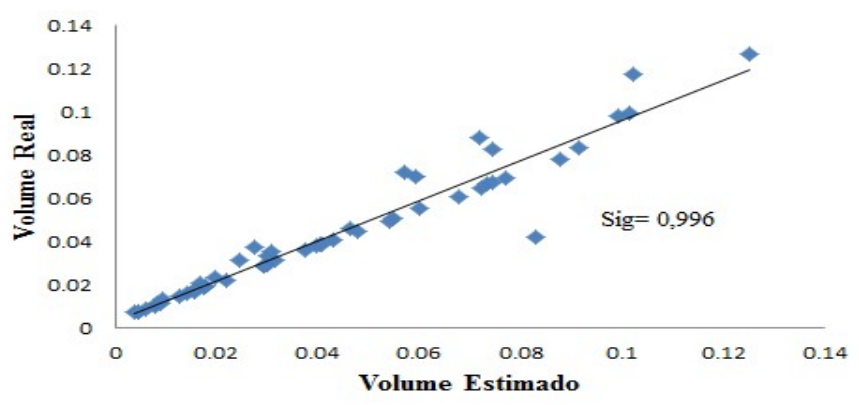

Figura 3 - Comparação entre valor real e o estimado com o modelo de melhor ajuste $\log V r t=-1,058+1,023 \log _{g t}+0,485 * 1 / \log _{g t}^{2}$ para o fuste

- Modelos de regressão de volume para os ramos

Os resultados de medição das diferentes variáveis dendrométricas dos ramos das 53 árvores de amostra que se derrubaram e mediram rigorosamente se calculou a matriz de correlação das variáveis para os ramos com o volume real total (Vrt) como variável dependente a respeito as variáveis independentes: $\mathrm{Db}$ diâmetro da base em cm; $\mathrm{D}_{0,30}$ diâmetro em $\mathrm{cm} ; \mathrm{D}_{1} \mathrm{~m}$ diâmetro em $\mathrm{cm} ; \mathrm{D}_{1 / 2}$ diâmetro em cm; Ht altura total em m; Gt área basal em $\mathrm{m}^{2}$ e Df diâmetro da parte mais fina do ramo em $\mathrm{cm}$. Os resultados da matriz de correlação que se mostram no Quadro 6 indicam que existe correlação com todas as variáveis, excepto o $D_{\mathrm{f}}$, sendo que a correlação mais forte é com $\mathrm{Gt}$, $\mathrm{d}_{0,30} \mathrm{e} \mathrm{d}_{1,30 \mathrm{~m}}$.

Quadro 6 - Matriz de correlação de Pearson das variáveis dendrométricas para os ramos de Brachystegia gossweilerii Hutch. \& Burt Davy

\begin{tabular}{|c|c|c|c|c|c|c|c|c|}
\hline & VrT & Db & D 0,30 & D1,30m & D1/2 & C total & Gt & Df \\
\hline VrT & 1 & $0,905^{* *}$ & $0,913^{* *}$ & $0,921^{* *}$ & $0,730^{* *}$ & $0,788^{* *}$ & $0,998^{* *}$ & $-.124^{\mathrm{ns}}$ \\
\hline $\mathrm{Db}$ & & 1 & $0,980^{* *}$ & $0,950^{* *}$ & $0,693^{* *}$ & $0,767^{* *}$ & $0,905^{* *}$ & $-.180^{\mathrm{ns}}$ \\
\hline $\mathrm{D} 0,30$ & & & 1 & $0,965^{* *}$ & $0,720^{* *}$ & $0,742^{* *}$ & $0,911^{* *}$ & $-.154^{\mathrm{ns}}$ \\
\hline $\mathrm{D} 1 \mathrm{~m}$ & & & & 1 & $0,719^{* *}$ & $0,709^{* *}$ & $0,919^{* *}$ & $-.161^{\mathrm{ns}}$ \\
\hline $\mathrm{D} 1 / 2$ & & & & & 1 & $0,581^{* *}$ & $0,724^{* *}$ & $-.105^{\mathrm{ns}}$ \\
\hline C total & & & & & & 1 & $0,784^{* *}$ & -.138 \\
\hline Gt & & & & & & & 1 & $-.122^{\mathrm{ns}}$ \\
\hline Df & & & & & & & & 1 \\
\hline
\end{tabular}

** A correlação é significativa a nível de 0,01 (bilateral).

ns Não significativa. 
No Quadro 7 são apresentados os modelos que melhor se ajustaram para os ramos, segundo as análises de bondade de ajuste e as capacidades preditivas, com o Vrt como variável dependente.

Quadro 7 - Melhores dez modelos testados para os ramos

\begin{tabular}{|c|c|c|c|c|c|c|c|}
\hline \multirow{2}{*}{$\mathbf{N}^{\mathbf{o}}$} & \multirow{2}{*}{ V. Ind. } & \multicolumn{6}{|c|}{ Modelos de melhor ajuste para fuste $\operatorname{com} V_{\text {rt }}$} \\
\hline & & $\mathbf{R}$ & $\mathbf{R}^{2}$ & Sx & REQM & DA & Equação \\
\hline 1 & Gt & 0,999 & 0,997 & 0,006 & 0,000 & 0,001 & $V r t=0,297 * g t^{1,987}$ \\
\hline 5 & Logdf & 0,995 & 0,997 & 0,048 & 0,000 & 0,024 & $\log V r t=1,476+0,063 \log d_{f}+\left(5,283 * 1 / \log d_{f}{ }^{2}\right)$ \\
\hline 3 & $\mathrm{Gt} ; \mathrm{Gt}^{05}$ & 0,995 & 0,997 & 0,138 & 0,001 & 0,000 & $\log V r t=0,000+0,301 g_{t}+0,098 g_{t}{ }^{0,5}$ \\
\hline 4 & Gt & 0,995 & 0,995 & 0,001 & 0,000 & 0,000 & $V r t=0,301+\left(0,000 * g_{t}\right)$ \\
\hline 5 & Logdf & 0,995 & 0,997 & 0,048 & 0,000 & 0,024 & $\log V r t=1,476+0,063 \log d_{f}+5,2831 / \log d_{f^{2}}$ \\
\hline 3 & $d^{2} 1 m$ & 0,976 & 0,953 & 0,004 & 0,000 & 0,000 & $\log V r t=1,476+0,063 \log d_{f}+\left(5,283 * 1 / \log d_{f}{ }^{2}\right)$ \\
\hline 7 & $\mathrm{~d}_{0,30^{\mathrm{al}}}$ & 0,920 & 0,959 & 0,077 & $-0,00$ & 0,005 & $V r t=0,005 * d_{0,30}^{0,908} h_{t}^{0,891}$ \\
\hline 7 & $\mathrm{~d} 1 \mathrm{~m}^{\mathrm{al}}$ & 0,959 & 0,979 & 0,049 & $-0,00$ & 0,003 & $V r t=0,009 * d 1 m^{1,815} h_{t}^{0,953}$ \\
\hline 9 & $\mathrm{~d}^{2} 1 \mathrm{mht}$ & 0,955 & 0,977 & 0,000 & $-0,00$ & 0,004 & $V r t=d^{2} 1 m\left(0,006+0,004 h_{t}\right.$ \\
\hline 9 & $\mathrm{~d}^{2} 0,30 \mathrm{~h}$ & 0,919 & 0,958 & 0,000 & $-0,00$ & 0,005 & $V r t=d^{2}{ }_{0,30}\left(0,008+0,003 h_{t}\right)$ \\
\hline
\end{tabular}

Mostra-se as equações de volume com os seus coeficientes, onde o modelo aritmético de melhor ajuste resultou ser o de Berkhout, representado com o número 1, cuja equação é: $V r t=0,297 * g t^{1,987}$,diferente do que melhor se ajustou para o fuste. Estes resultados diferem de LORES (2012) que encontrou o modelo aritmético de Schumacher e Hall.

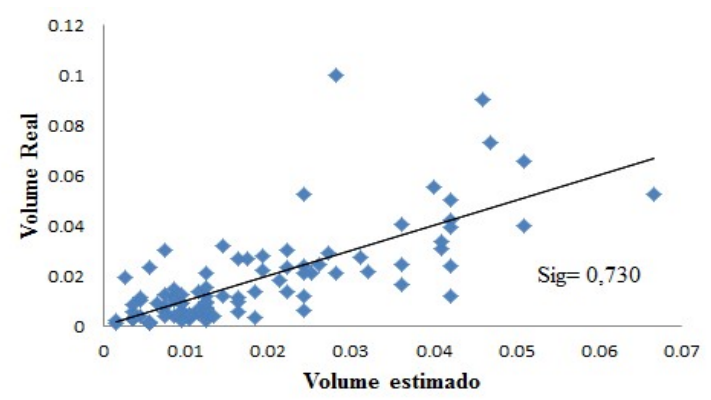

Figura 4 - Comparação entre valor real e o estimado com o modelo de melhor ajuste $\left(\mathrm{Vrt}=0,297 \mathrm{Gt}^{0,987}\right)$ para os ramos 
70 Ndjamba, A., et al.

\section{Conclusões}

Os modelos que melhor se ajustaram, pela confiabilidade estatística, indicados para a construção de tabelas de volume do fuste e ramos, nas condições do Miombo da aldeia de Calombo são: o modelo aritmético representado com $\mathrm{o}$ número 5 na lista dos modelos $\log v=b_{0}+b_{1} \log X+b_{2} 1 / \log \left(X^{2}\right)$, isto é, para o fuste e o representado com o número 1 cuja equação é $V r t=b_{0} X^{b 1}$, para os ramos.

\section{Bibliografia}

ARES, A.E., 1999. Tablas Dasométricas para bosques naturales de Pinus tropicalis Morelet para la EFI La Palma. $94 \mathrm{~h}$. Tesis (en opción al grado científico de Doctor en Ciencias Forestales). Universidad de Pinar del Rio.

CAILLIEZ, F., 1980. Estimación del Volumen forestal y predicción del rendimento: Estimación del Volumen. Estudio FAO. Montes. Roma, $91 \mathrm{pp}$.

COBAS, O., 2013. Tablas dendrométrica y dasométricas de Carapa Guianensis Aubl. en bosques pluvisilvas de montaña de Baracoa, provincia Guantánamo, pp 29-68.

DINIZ, A.C., 2006. Características mesológicas de Angola. 2a Ed. Lisboa, Instituto Português de Apoio al Desarrollo. ISBN 972-8975-02-3.

FREIRE, E.F.V.D., 2010. Estudo e avaliação da caça e preservação da flora e fauna de Angola. $57 \mathrm{pp}$.

JAY, N.F.P., 2014. Eficiência do Método de Bitterlich na Avaliação de Parâmetros Dasométricos na Formação Florestal do Miombo, pp. 7-38.

LEITE, F.S., RESENDE, A.V., 2010. Estimativa do volume de madeira partindo do diâmetro da cepa em uma área explorada de floresta amazónica de terra firme. Revista Ciência Florestal, Santa Maria, 20(1): 69-79.

LOETSCH, F., 1973. Forest Inventory: Munich. BLV Verlagsgesellschaft München. 2 ed., Vol. II, 469 pp.

LORES, Y., 2012. Tablas dendrométricas y dasométricas de Calophyllum antillanum Britton, Carapa guianensis Aubl. y Andira inermis Sw. en bosques pluvisilvas de montaña de Baracoa, provincia Guantánamo. 99 h. Tesis (en opción al grado científico de Doctor en Ciencias Forestales). Universidad de Pinar del Rio. 79 pp.

MACHADO, S.A., 2003. Dendrometria. Curitiba, 309 pp.

MALENGUE, A.H., 2014. Avaliação do potencial de produtos florestais não madeireiros na área florestal de Calombo, Cuíma. FCA.

MINADER, 2011. Política Nacional de Florestas, Fauna Selvagem e Áreas de Conservação. Segunda Versão, pp. 4-56. 
PADILLA, G., 1999. Tablas dasométricas para plantaciones de Pinus tropicalis Morelet. $90 \mathrm{~h}$. Tesis (en opción al grado científico de Doctor en Ciencias Forestales). Universidad de Pinar del Rio. 49pp.

PEÑALVER, A., 1991. Estudio de crecimiento y rendimiento de las plantaciones de Eucalyptus sp. de la provincia de Pinar del Río. 101 h. Tesis (en opción al grado científico de Doctor en Ciencias Forestales). UPR. !Hermanos Saiz Montes de Oca".

PRODAN, M., PETERS, R., COX, F., REAL, P., 1997. Mensura Forestal. Serie de Investigación y Educación en Desarrollo Sostenible. San José, Costa Rica. 561 pp.

SALAS, C., 2002. Ajuste y validación de ecuaciones de volumen para un relicto del bosque de Roble-Laurel-Lingue. Bosque 23(2): 81-92.

SPURR, S.H., 1952. Forest inventory. New York, Ronald Press, 476 pp.

THOMAS, C., ANDRADE, C.M., SCHNEIDER, P.R., FINGER, C.A.J., 2016. Comparação de equações volumétricas ajustadas com dados de cubagem e análise de tronco. Ciência Floresta, Vol. 16: 319-327.

ZALDÍVAR, A., 2001. Tablas dasométricas para plantaciones de Hibiscus elatus en la provincia de pinar del Río. 100 h. Tesis (en opción al grado científico de Doctor en Ciencias Forestales). Universidad de Pinar del Rio. 